\title{
Diagnostic efficacy of panoramic mandibular index to identify postmenopausal women with low bone mineral densities.
}

\author{
B.K. Yashoda Devi ${ }^{1}$, Nagaraju Rakesh ${ }^{2}$, Nagi Ravleen ${ }^{3}$ \\ ${ }^{1}$ Professor \&HOD. Dept of Oral Medicine, Diagnosis and Radiology. M. S. Ramaiah Dental College \& Hospital, Msrit Post, \\ New Bel Road, Bangalore-560054, Karnataka ( India). \\ ${ }^{2}$ Associate Professor. Dept of Oral Medicine, Diagnosis and Radiology. M. S. Ramaiah Dental College \& Hospital, Msrit Post, \\ New Bel Road, Bangalore-560054, Karnataka ( India). \\ ${ }^{3}$ PG Student. Dept of Oral Medicine, Diagnosis and Radiology. M. S. Ramaiah Dental College \& Hospital, Msrit Post, New Bel \\ Road, Bangalore-560054, Karnataka ( India).
}

\author{
Correspondence: \\ Dept of Oral Medicine, Diagnosis and Radiology \\ M.S.Ramaiah Dental College \& Hospital, \\ Msrit Post, New Bel Road, \\ Bangalore-560054 \\ Karnataka (India) \\ Email:dryashodadevi@yahoo.com
}

Received: $18 / 07 / 2011$

Accepted: 02/10/2011
Yashoda Devi B.K., Rakesh N., Ravleen N. Diagnostic efficacy of panoramic mandibular index to identify postmenopausal women with low bone mineral densities. J Clin Exp Dent. 2011;3(5):e456-61.

http://www.medicinaoral.com/odo/volumenes/v3i5/jcedv3i5p456.pdf

\begin{abstract}
Objectives: The aim of the study was to compare and assess the accuracy of panoramic mandibular index (PMI) and antegonial index (AI) in the panoramic radiographs of postmenopausal women with normal and low skeletal bone mineral densities( BMD) diagnosed by using dual energy x-ray absorptiometry ( DXA).

Study Design: In panoramic radiographs obtained from 40 post menopausal women( 20 normal and 20 osteoporotic) aged between 50-75 who's BMD has already been assessed by a DXA, the mean was calculated for PMI and AI index values measured in the right and left mandibles. The PMI and AI index values were evaluated using the student's $t$ test. The correlation between the observers for indices was calculated by Pearson correlation coefficient test.

Results: A statistically difference was shown between the PMI values in the osteoporotic group and normal group $(\mathrm{t}=13.280, \mathrm{p}<0.001)$; however, AI showed no significant difference between the groups. For PMI, the area under the receiver operating curve (ROC) analysis in identifying women with low BMD was 0.821 ( Standard error: SE $=0.0676), 95 \%$ confidence interval $(\mathrm{Cl})=0.668$ to 0.924$)$ which was moderately accurate.

Conclusion: PMI values were found to be smaller among female patients with osteoporosis, compared with normal patients in the present study. Our results suggest that PMI has a better efficacy in identifying postmenopausal women with osteoporosis in comparison with AI.
\end{abstract}

Key Words: menopause, osteoporosis, dual-energy X-ray absorptiometry, panoramic radiography, panoramic mandibular index, antegonial index. 


\section{Introduction}

Osteoporosis is one of the most prevalent conditions associated with aging and is thus a major public health problem (1). It is characterized by compromised bone strength, is frequently not detected until a fracture occurs and is considered to be a silent disease that entails significant social and economic burdens $(2,3)$. It is known that after the age of thirty five the bone mineral density (BMD) of men and women gradually decreases with increasing age. Women tend to lose BMD more rapidly than men, especially after the menopause. As a result osteoporosis is three times more common among women than men (4). Osteoporosis is defined as a "skeletal disorder characterized by low bone mass and micro architectural deterioration of bone tissue leading to enhanced bone fragility, with consequent increase in fracture risk" (5).

Osteoporosis is usually diagnosed by BMD measurements, expressed as a T- score. According to World Health Organization (WHO) criteria, BMD values are divided into following clinical guidelines: normal ( $\mathrm{T}$ -score $\geq-1.0$ ), osteopenia (T-score between -1.0 and -2.5 ), and osteoporosis (T-score $\leq-2.5$ ) (2). Osteoporotic fractures are associated with substantial morbidity and high mortality risk involving spine, hip, forearm and proximal humerus and among them hip fractures incur the greatest morbidity and mortality, and give rise to the highest direct costs for health services $(5,6)$. Single- or dual-photon absorptiometry, quantitative computed tomography (QCT), single- or dual-energy X-ray absorptiometry (DXA) and quantitative ultrasound are among the techniques developed to identify individuals with skeletal osteoporosis. DXA is used extensively around the world as it has a high reliability in identifying individuals with low BMD and delivers a low radiation dose (7). However; BMD testing for all postmenopausal women is not practicable in many countries where a bone assessment method, especially DXA, is not widely available. Moreover, most of the post- menopausal women have more opportunities to visit dental clinic for the treatment of dental caries and periodontal disease than to visit a medical office for diagnosis of osteoporosis prior to fracture $(8,9)$. Hence the panoramic radiograph plays a vital role in identifying post menopausal women with undetected osteoporosis. The effect of osteoporosis on the jaw bones has been studied intensively. Cortical indices of the mandible on panoramic radiographs, such as cortical width and cortical shape, are significantly associated with BMD of the general skeleton, and risk of osteoporotic fractures in postmenopausal women (2).

In the present study, PMI and AI indices on the panoramic radiographs were used for the diagnosis of osteoporosis in postmenopausal women and the aim of the study was to compare and assess the accuracy of PMI and $\mathrm{AI}$ in the panoramic radiographs of postmenopausal women with normal and low skeletal bone mineral densities diagnosed by using DXA.

\section{Materials and methods \\ Sample}

This study was approved by the Ethics Committee of the Institution and the subject's informed consent was also obtained after explaining of the general nature of the study. 40 post-menopausal women with ages ranging from 50-75 who's BMD has already been assessed by DXA in M. S. Ramaiah Memorial Hospital, Bangalore had undergone panoramic radiographic examination in $\mathrm{M}$. S. Ramaiah Dental College, Bangalore. They were divided into control group with normal skeletal BMD ( $\mathrm{n}=$ 20 ; mean age $65.30 \pm 5.430$ ), and osteoporotic group with low skeletal BMD $(n=20$; mean age $70.15 \pm 3.422)$. Subjects included for the study were postmenopausal women with no history of menstruation for at least five years and the patient was ambulatory for participating in the study. Exclusion criteria was (1) postmenopausal women whose precise medical histories could not be confirmed by hospital records, patients with metabolic bone disease (hyperthyroidism, hyperparathyroidism, Paget disease, osteomalacia), diabetes mellitus and mandibular bone destruction; (2) patient had a history of malignancy $\leq 5$ years prior to signing informed consent, except for adequately treated basal cell or squamous cell carcinoma or in situ cervical cancer; (3) patients with significant renal impairment and liver disorders (4) patients with history of smoking, tobacco use and use of drugs that affect bone metabolism such as estrogen, oral bisphosphonates, systemic glucocorticoids within the prior 6 months.

BMD Assessment

BMD at the proximal femur (femoral neck) was determined by DXA in M.S. Ramaiah Memorial Hospital, Bangalore. Height and weight was measured at the time of DXA measurement. Body Mass Index (BMI) was calculated from the measured height and weight of the patient by using weight/square of height $\left(\mathrm{kg} / \mathrm{m}^{2}\right)$. The BMD scores were given as the ' $T$ ' score according to WHO classification (2) of osteoporosis. Normal BMD was defined as T- score of -1.0 or more and osteoporosis was defined as a BMD T- score of -2.5 or less, according to the WHO classification.

\section{Conventional panoramic radiographic examination}

Panoramic radiographs were taken for all patients who gave informed consent at time of DXA measurement with panoramic X ray machine (PLANMECA, cc Proline - 2002).In order to achieve a standard, the radiographs were obtained by a single examiner at $10 \mathrm{~mA}$ and $18 \mathrm{sec}$; the voltage varied between $60 \mathrm{kVp}-75 \mathrm{kVp}$ depending on the age, sex and built of the patient. Patients were positioned in the dental panoramic machine in such a way that the vertical line produced by the machine was alig- 


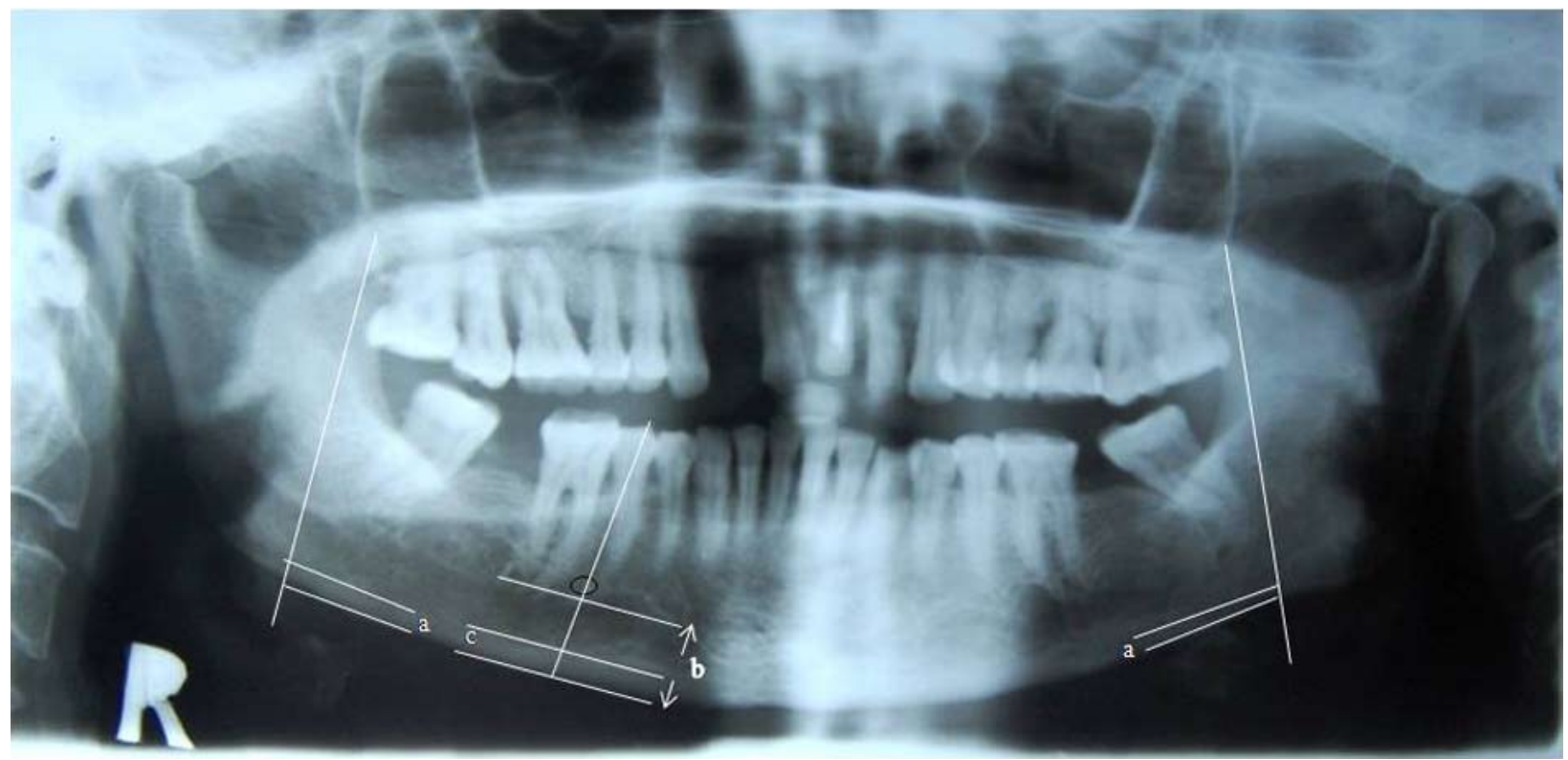

Fig.1. Measurement of the panoramic mandibular index(PMI) $[c / b]$ and the antegonial index( AI) [a] used in the study

ned with the patient's sagittal plane, with the horizontal line ( Frankfort plane) parallel to the floor. All the exposed panoramic films were processed manually. After air drying, the radiographs were viewed and evaluated on a viewing box under normal operating illumination. Two observers independently measured PMI and AI index manually, using a x 6 magnifying loupe, caliper and a plastic millimetre $(\mathrm{mm})$ ruler. All measurements were made with the observers blinded to the reference osteoporotic diagnosis.

The inferior PMI is the ratio of the thickness of the mandibular cortex to the distance between the inferior margin of the mental foramen and the inferior mandibular cortex $(10,11)$. (Fig.1). It was measured bilaterally at the site of mental foramen with the help of callipers and the mean of the measurements was used as the exposure measure in the analysis. AI is the measurement of the cortical width in the region anterior to the gonion at a point by extending a "best fit" line along the anterior border from the ascending ramus down to the lower border of the mandible. $(2,10)$ Measurements were made separately on the right and left mandibular sides and their means were calculated. (Fig.1).

\section{Statistical analysis}

The Microsoft Excel and SPSS (SPSS Inc, Chicago) software packages were used for data entry and analysis. Data was presented as mean values, standard error of means (SEM), numbers and percentages. The student ${ }^{6} t$ ' test was used to determine whether there was a statistical difference between groups in the parameters measured. The correlation between the observers was calculated by Pearson correlation coefficient test. Receiver operating characteristic (ROC) curve analysis and threshold values were used to determine the sensitivity, specificity, and likelihood ratio of PMI in identifying women with low BMD. Area under the ROC curve (AUC) was also calculated. According to arbitrary guideline (12), we distinguished between non informative $(\mathrm{AUC}=0.5)$, less accurate $(0.5<$ AUC $<0.7)$, moderately accurate $(0.7<$ AUC $<0.9)$, highly accurate $(0.9<$ AUC $<0.9)$, perfect tests $(\mathrm{AUC}=1)$. In the entire above tests a "p" value of less than 0.05 was accepted as indicating statistical significance.

\section{Results}

The mean BMI $\left(\mathrm{kg} / \mathrm{m}^{2}\right)$ was $28.37 \pm 4.71$ and 23.67 \pm 4.02 for the normal and osteoporotic group, respectively $(\mathrm{p}<0.05)$. (Table 1$)$ The mean values of PMI were $0.329 \pm 0.084$ for normal and $0.221 \pm 0.102$ for the os-

\begin{tabular}{|l|l|l|l|l|l|l|l|l|}
\hline Group & $\mathbf{n}$ & $\begin{array}{l}\text { Mean } \\
\text { BMI }\end{array}$ & Standard Deviation & Median & Minimum & Maximum & $\begin{array}{l}\text { 't' } \\
\text { value }\end{array}$ & $\begin{array}{l}\text { 'p' } \\
\text { value }\end{array}$ \\
\cline { 1 - 6 } Normal & 20 & 28.375 & 4.7146 & 28.050 & 19.8 & 41.0 & \multirow{2}{*}{11.496} & \multirow{2}{*}{$0.002^{*}$} \\
\cline { 1 - 7 } Osteoporotic & 20 & 23.675 & 4.0252 & 23.150 & 16.6 & 31.2 & & \\
\hline
\end{tabular}

BMI: body mass index, $n$ : number

Table 1. Body mass index $\left(\mathrm{kg} / \mathrm{m}^{2}\right)$. *Statistically significant difference between the groups 


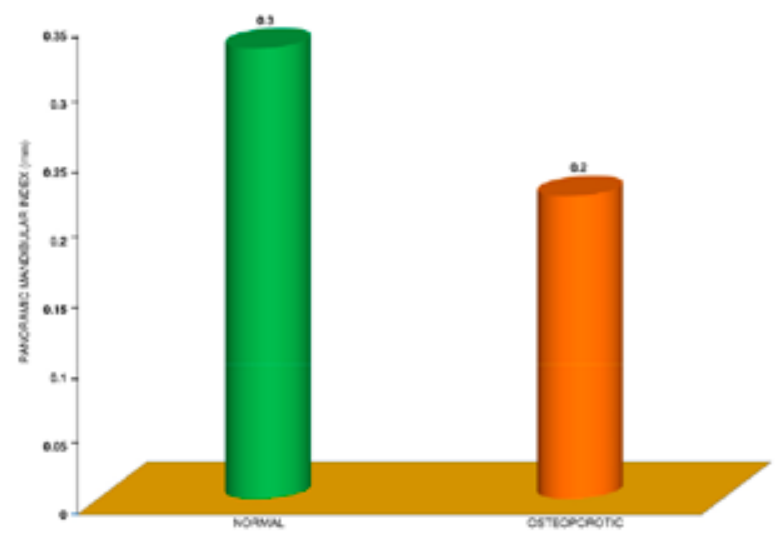

Fig.2. Mean values for panoramic mandibular index between the groups. ${ }^{*} p=<0.001$, " $t$ " test, indicates a significant decrease in relation to osteoporotic group

teoporotic group. (Fig.2) A statistically difference was shown between the PMI values in the osteoporotic group and normal group $(t=13.280, p<0.001)$. (Table 2$)$. The mean values of AI were $2.388 \pm 0.599$ and $2.000 \pm 0.739$ for normal and the osteoporotic group, respectively but no statistically significant difference was observed between the groups in the study $(\mathrm{p}>0.05)$. (Table 3 )

For PMI, the area under the ROC curve (AUC) analysis in identifying women with low BMD was $0.821(95 \% \mathrm{Cl}$ $=0.668$ to 0.924$)$. The threshold value that provided the highest validity (minimal false negative and false positive results) corresponded to PMI of $<0.25 \mathrm{~mm}$ which provided a sensitivity of $85 \%(95 \% \mathrm{Cl}=62.1 \%-96.6$ $\%)$, specificity of $80 \%(95 \% \mathrm{Cl}=56.3 \%-94.1 \%)$, and likelihood ratio of $4.25(95 \% \mathrm{Cl}=3.2-5.7)$.(Fig. 3$)$.

In the present study there was statistically significant correlation between the observers in measuring the PMI and AI. For PMI, it was $(r=0.961, p<0.001)$ and $(r=$ $0.936, \mathrm{p}<0.001$ ), normal and the osteoporotic group respectively. For AI, the correlation was $(r=0.974, p$ $<0.001)$ for the normal and $(r=0.984, p<0.001)$ for the osteoporotic group.

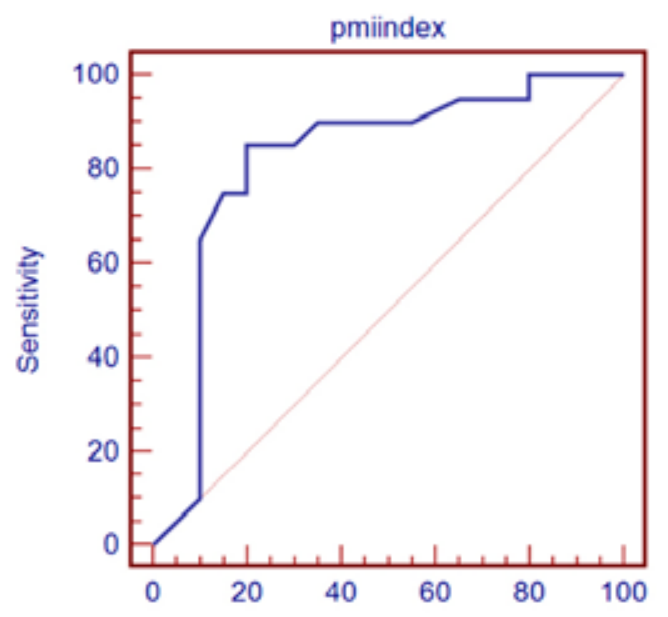

Fig.3. Receiver operating characteristic (ROC) curve for identifying postmenopausal women with osteoporosis by panoramic mandibular index.

\section{Discussion}

Osteoporosis, a generalized reduction in the amount of bone tissue, is a major public health problem for middle - aged and elderly patients. Bone loss occurs with age in men and women (age -related osteoporosis), but in the latter the rate of loss increases at the time of menopause (postmenopausal osteoporosis) (1). This reduction in bone tissue is associated with an increased risk of fractures, pain and consequently morbidity for patients $(1,8,13)$. Osteoporosis is also influenced by BMI of the women. The WHO (14) classified BMI as follows: Normal weight $18.5 \geq \mathrm{BMI}<25$, overweight $25 \geq \mathrm{BMI}<30$ and obese $\mathrm{BMI} \geq 30$. It was reported that $\mathrm{BMI}$ significantly correlates with BMD and people with a BMI of $20-25 \mathrm{Kg} \mathrm{m}^{-2}$ have a higher rate of bone loss than those who are heavier. Hence, according to WHO criteria, in the present study the mean BMI of osteoporotic group $(23.67 \pm 4.02)$ was less compared to controls $(28.37 \pm$ $4.71)$ with $p$ value $<0.05$, indicating greater susceptibility to osteoporotic fractures. Since the disease is preventable, diagnostic techniques are of major importance (14). Hence, considerable effort has been expended in

\begin{tabular}{|l|l|l|l|l|l|l|l|l|}
\hline Group & $\mathbf{n}$ & Mean & Standard Deviation & Median & Minimum & Maximum & 't' value & 'p' value \\
\hline Normal & 20 & 0.329 & 0.084 & 0.335 & 0.14 & 0.50 & \multirow{2}{*}{13.280} & $<0.001^{*}$ \\
\cline { 1 - 8 } Osteoporotic & 20 & 0.221 & 0.102 & 0.190 & 0.11 & 0.50 & & \\
\hline
\end{tabular}

Table 2. Panoramic mandibular index values. *Statistically significant difference between the groups

\begin{tabular}{|l|l|l|l|l|l|l|l|l|}
\hline Group & $\mathbf{n}$ & Mean & Standard Deviation & Median & Minimum & Maximum & 't' value & 'p' value \\
\hline Normal & 20 & 2.388 & 0.599 & 2.250 & 1.50 & 3.75 & \multirow{2}{*}{3.320} & \multirow{2}{*}{.076} \\
\hline Osteoporotic & 20 & 2.000 & 0.739 & 2.000 & 1.00 & 4.00 & & \\
\hline
\end{tabular}

Table 3. Antegonial index values. No significant difference between the groups 
identifying methods of detecting individuals with osteoporosis at an early age (1).

BMD in the mandible has been shown, in a number of studies, to be positively correlated with that in lumbar spine, femoral neck and forearm, important sites in osteoporosis (15). Identification of the signs of osteoporosis in dental panoramic radiographs is crucial in the diagnosis of this condition (7). Several panoramic radiographic measurements, also known as panoramic radio morphometric indices, have been developed to assess cortical shape and width, either qualitatively $(16,17)$ or quantitatively $(11,18)$. Results of the studies suggested that the thickness of mandibular cortical bone is decreased in osteoporotic patients and inferior border of the mandible is more porous than the controls. The present study compares the PMI and AI index values of female patients with osteoporosis and a control group using panoramic radiography. The study has also used DXA for the measurement of BMD in the femoral neck which is considered as a reference standard examination for BMD assessment and the best predictor of fracture risk (2).

PMI was first proposed by Benson et al (1991) (18) as radio morphometric index of adult cortical bone mass. The recent studies in the literature $(1,2)$ on PMI values have been performed mostly on women. As an average, the results of these literatures reveal PMI values between $0.31-0.38$ for women. In a study by Horner and Devlin (1998) (1), the PMI values measured in females with osteoporosis on panoramic radiographs were compared with the mandibular bone values measured with DXA, and they found a significant relation between the two. Studies, measuring PMI values found that the values that decrease with increasing age are smaller among white individuals than among black individuals and among women compared with men. They are also smaller in female patients with osteoporosis than in healthy female individuals; this was found statistically significant in the present study at $p$ value $<0.001(5,7)$.

We also calculated the sensitivities and specificity at a number of threshold to prepare ROC curve and the area under the ROC curve (AUC) was also calculated. AUC for identifying postmenopausal women with osteoporosis by PMI of the mandible was $0.821(95 \% \mathrm{Cl}, 0.668$ -0.924 ) which indicates moderate accuracy for PMI and the optimal cut off point was $<0.25 \mathrm{~mm}$ for osteoporosis in the present study. The results for osteoporosis diagnosis were similar to those found by other authors (2, 5) which suggest that PMI is a useful screening tool to detect osteoporosis.

In this study, the AI had poor reliability, and no statistically, significant differences were observed in mean values among the BMD groups. Various studies have suggested positive correlation between AI and BMD. Léger ton, et al (1999) (11) evaluated AI in 500 panoramic radiographs in terms of age, sex dentition and social class, found a negative correlation with age, and this was interpreted as a possible indictor of skeletal osteopenia. A study of 52 edentulous females found that AI values measured on panoramic films were lower in the group with osteoporosis, and antegonial region measurements are a useful method to identify osteoporosis risk groups (19). In contrast to the above studies, Leite FA, et al (2010) (2) measured AI in panoramic radiographs of 351 postmenopausal women with skeletal osteoporosis and found negative correlation between AI and BMD. This result was comparable to the present study suggesting that AI measurements cannot be used to identify postmenopausal women with undetected low skeletal BMD or osteoporosis.

Panoramic radiographs measurements are associated with intrinsic errors and observer variability. There is limitation in the repeatability of panoramic radiographic measurements (14). Yasar F, et al ( 2006) (14) found that number of mandibular teeth had the highest repeatability $(r=1$; SD: 0,000$), \mathrm{M} / \mathrm{M}$ ratio $(r=0.864$; coefficient of repeatability : 0.65$)$, PMI ( $r=0.871$; coefficient of repeatability:0.65), were following in decreasing order and cortical width $(\mathrm{r}=0.069$; coefficient of repeatability : 1.17) had the worst repeatability. The linear measurements have greater observer dependency, therefore to obtain the same results in repeated observations, the borders of the mental foramen should be determined correctly and later the necessary drawings should be made and finally these configurations should coincide with each other. That is, as the operations that must be performed for measuring index increases, the measurement error would also increase, so it might be more effective to use simple indexes to reduce measurement error and increase agreement between the repeated measurements (20). Although there are published data about the problems with repeatability of panoramic radio morphometric indices $(2,21)$, most authors conclude that the efficacy of these indices in diagnosing osteoporosis is sufficient $(10,17)$. The repeatability of PMI and AI was evaluated by Pearson correlation coefficient in the present study, and the correlation between the observers for PMI ( normal $: r=0.961$, osteoporotic $: r=0.936$ ) and AI( normal: $r=0.974$, osteoporotic $: r=0.984$ ) was found to be statistically significant . Our results demonstrate that PMI has better efficacy than the AI to screen and refer patients with positive findings for further assessment for the potential diagnosis of osteoporosis.

\section{Conclusion}

Results of the study suggest that elderly women with PMI $<0.25 \mathrm{~mm}$ in the panoramic radiographs should be referred for advanced osteoporosis investigation. Although radio morphometric indices are regarded as an ancillary method for the diagnosis of osteoporosis on panoramic radiographs, they should be a routine proce- 
dure in dental examination and dentists may be able to refer postmenopausal women younger than 65 years for bone densitometry on the basis of incidental findings on dental panoramic radiographs.

\section{Acknowledgement}

We would like to thank our Principal and Professor Dr. H. N. Shama Rao for his support and guidance.

\section{References}

1. Horner K, Devlin H. The relationship between mandibular bone mineral density and panoramic radiographic measurements. J Dent. 1998; 26: 337-343.

2. Leite AF, Figueiredo PT, Guia CM, Melo NS, Paula APD. Correlations between seven panoramic radiomorphometric indices and bone mineral density in postmenopausal women. Oral Surg Oral Ned Oral Pathl Oral Radiol Endod. 2010; 109:449-456.

3. Atik OS, Gunal I, Korkusuz F. Burden of osteoporosis. Clin Orthop Relat Res. 2006; 443: 19-24.

4. Verheij JGC, Geraets WGM, Vander Stelt PF, Horner K, Lindh C, Nicopoulou KK, et al. Prediction of osteoporosis with dental radiographs and age. Dentomaxillofac Radiol. 2009; 38: 431-7.

5. Gulsahi A, Yuzugullu B, Imirzalioglu P, Genc Y. Assessment of panoramic radiomorphometric indices in Turkish patients of different age groups, gender and dental status. Dentomaxillofac Radiol. 2008; 37:288-292.

6. Nakamoto T, Taguchi A, Ohtsuka M, Suei Y, Fujita M, Tanimoto K, et al. Dental panoramic radiograph as a tool to detect postmenopausal women with low bone mineral density: untrained general dental practitioners, diagnostic performance. Osteoporosis Int. 2003; 14: 659-664.

7. Dagistan S, Bilge OM. Comparison of antegonial index, mental index, panoramic mandibular index and mandibular cortical index values in the panoramic radiographs of normal males and male patients with osteoporosis. Dentomaxillofac Radiol. 2010; 39: 290-4.

8. Ishii K, Taguchi A, Nakamoto T, Ohtsuka M, Sutthiprapaporn P, Tsuda M, et al . Diagnostic efficacy of alveolar bone loss of the mandible for identifying postmenopausal with femoral osteoporosis. Dentomaxillofac Radiol. 2007; 36: 28-33.

9. Taguchi A, Ohtsuka M, Tsuda M, Nakamoto T, Kodama I, Inagaki $\mathrm{K}$, et al. Risk of vertebral osteoporosis in post-menopausal women with alterations of the mandible. Dentomaxillofac Radiol. 2007; 36: 143-8.

10. Gulsahi A, Paksoy CS, Ozden S, Kucuk NO, Cebeci ARI, Genc Y. Assessment of bone mineral density in the jaws and its relationship to radiomorphometric indices. Dentomaxillofac Radiol. 2010; 39: 284-289.

11. Ledgerton D, Horner K, Devlin H, Worthington H. Radiomorphometric indices of the mandible in a British female population. Dentomaxillofac Radiol. 1999; 28: 173-181.

12. Swets JA. Measuring the accuracy of diagnostic systems. Sci. 1998; 240: 1285-1293.

13. Sugiura M, Nakamura M, Ogawa K, Ikoma Y, Ando F, Yano M. Bone mineral density in post- menopausal female subjects is associated with serum antioxidant carotenoids. Osteoporosis Int. 2008; 19: 211-19.

14. Yasar F, Akgunlu F. The differences in panoramic mandibular indices and fractal dimension between patients with and without spinal osteoporosis. Dentomaxillofac Radiol. 2006; 35: 1-9.

15. Horner K, Devlin H, Alsop CW, Hodgkinson IM, Adams JE. Mandibular bone mineral density as a predictor of skeletal osteoporosis. Br J Radiol. 1996; 69:1019-1025.

16. Lee K, Taguchi A, Ishii K, Suei Y, Fujita M, Nakamoto T et al. Visual assessment of the mandibular cortex on panoramic radiographs to identify postmenopausal women with low bone mineral densities. Oral Surg Oral Med Oral Path Oral Radiol Endod. 2005;
100:226-31.

17. Klemetti E, Kolmakov S, Kroger H. Pantomography in assessment of the osteoporosis risk group. Scand J Dent Res. 1994; 102: 6872 .

18. Benson BW, Prihoda TJ, Glass BJ. Variations in adult cortical bone mass as measured by a panoramic mandibular index. Oral Surg Oral Med Oral Path. 1991; 71:349-356.

19. Dutra V, Devlin H, Susin C, Yang J, Horner K, Fernandes ARC. Mandibular morphological changes in low bone mass edentulous females: evaluation of panoramic radiographs. Oral Surg Oral Med Oral Pathol Oral Radiol Endod. 2006; 102: 663-8.

20. Landis JR, Koch GG. The measurement of observer agreement for categorical data. Biometrics. 1977; 33:159-174.

21. Drozdzowska B, Pluskiewicz W, Tarnawska B. Panoramic - based mandibular indices in relation to mandibular bone mineral density and skeletal status assessed by dual energy X- ray absorptiometry and quantitative ultrasound. Dentomaxillofac Radiol. 2002; 31:361-7. 